\title{
A Learning Multiple-Valued Logic Network that can Explain Reasoning
}

\author{
Non-member Zheng Tang \\ (Miyazaki University) \\ Non-member Okihiko Ishizuka (Miyazaki University) \\ Non-member Koichi Tanno (Miyazaki University)
}

\begin{abstract}
This paper describes a learning multiple-valued logic (MVL) network that can explain reasoning. The learning MVL network is derived directly from a canonical realization of MVL functions and therefore its functional completeness is guaranteed. We develop traditional back-propagation to the MVL networks and drive a specific algorithm for the MVL networks. The algorithm combines back-propagation learning with other features of MVL networks, including the prior human knowledge on the MVL networks, for example, the architecture, the number of hidden units and layers, and many other useful parameters for the networks. The prior knowledge from the MVL canonical form can be used as initial parameters of the learning MVL network in its learning process. As a result, the prior knowledge can guide the back-propagation learning process to get started from a point in the parameter space that is not far from the optimal one, thus, back-propagation can fine-tune the prior knowledge for achieving a desired output easily. This cooperative relation between the prior knowledge and the back-propagation learning process is not always present in neural networks. The learning process in the MVL network also shares some cytology behaviors, in particular the cell adhesion, the cell aptopsis (the death of cell), and the cluster cell aptopsis (the death of cluster cells), and presents these properties in the artificial MVL network successfully. Simulation results are also given to confirm the effectiveness of the methods.
\end{abstract}

Keywords: Multiple-valued logic, Learning, Back-propagation, Reasoning

\section{Introduction}

Besides reductions in interconnection and chip area, multiple-valued logic (MVL) networks have emerged as powerful image processing ${ }^{[1]}$ and speech recognition ${ }^{[2]}$ techniques. However, these networks cannot learn or adapt to gradual change in their environments. By adding learning and adaptation ability in these techniques, we can design systems that can adjust to environment changes and get their power from their ability to learn, and, on the other hand, from their capacity to be modified and extended. Learning is also necessary when the information about inputs/outputs is unknown, so that no design of a network can be performed in advance. Furthermore, the ability to adapt and continue learning is also essential in many areas such as speech recognition where training data is limited and new talkers, new words, new dialects, new phrases, and new environments are continuously encountered. Several MVL networks with learning capability have been proposed ${ }^{[3-8]}$. These learning MVL networks, like conventional neural networks require arbitrary choices of the number of hidden units and layers. The learning is performed either in a "black box " without any prior knowledge about networks ${ }^{[3,5]}$ or in binary versions ${ }^{[6-8]}$ by converting multiple-valued to binary, and then performing the learning as the usual neural networks. This paper describes a new learning MVL network that can explain reasoning. The key idea is to make a widest possible use of the prior knowledge we have on MVL networks while constructing a MVL network, and conducting the learning in a manner analogous to neural back-propagation. The prior knowledge, even sometimes they are incomplete, can guide neural back-propagation learning process to get started from a point in the parameter space that is not far from the optimal one. The learning capability of the MVL networks is confirmed through simulations. Further, the learning for the MVL networks we are studying shares some biological cytology properties, such as the cell adhesion, the cell aptopsis (the death of cell) and the cluster cell aptopsis (the death of cluster cells) and shows these behaviors in simulations of the artificial MVL networks.

\section{MVL Networks}

A multiple-valued logic, begun with Post ${ }^{[9]}$ and continued with many further developments [10].[11], is a set which extends binary notation to $0,1,2, \ldots,(m-2)$, $(m-1)$, a set of $m$ values. Post algebra consists of a set $Q$ of $m$ logic values and a set $V$ of three basic operations : MAX $\left(x_{1}, x_{2}, \ldots, x_{n}\right), M I N\left(x_{1}, x_{2}, \ldots, x_{n}\right)$ and literal, denoted by,$+ \cdot$, and $x(a, b)$ respectively.

$$
\begin{aligned}
M & =(Q, V) \\
Q & =\{0,1,2, \ldots, m-1\}, 0<1<\ldots<m-1 \\
V & =\{+, \cdot, x(a, b)\}
\end{aligned}
$$

Let $x_{1}, x_{2}, \ldots, x_{n}$ to be the $n$ multiple-valued variables of $Q$. It is well known that any MVL function can 
Table 1. Example 1 of a quaternary function.

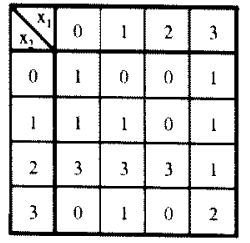

be realized in a sum-of-products form [12],[13] :

$$
\begin{aligned}
F\left(x_{1}, x_{2}, \cdots x_{n}\right)= & \sum F\left(e_{1}, e_{2}, \cdots e_{n}\right) \cdot x_{1}\left(e_{1}, e_{1}\right) \\
& \cdot x_{2}\left(e_{2}, e_{2}\right) \cdots x_{n}\left(e_{n}, e_{n}\right) \cdot(1)
\end{aligned}
$$

where $x_{1}, x_{2}, \cdots, x_{n}$ are $n$ multiple-valued variables, $e_{i}$ $\in 0,1,2, \cdots,(m-1), i=1,2, \cdots, n$ and

$$
\begin{aligned}
x_{1}+x_{2}+\cdots+x_{n}= & M A X\left(x_{1}, x_{2}, \cdots, x_{n}\right) \\
= & \text { the largest value of } \\
& \left(x_{1}, x_{2}, \cdots, x_{n}\right) \\
x_{1} \cdot x_{2} \cdots x_{n}= & M I N\left(x_{1}, x_{2}, \cdots, x_{n}\right) \\
= & \text { the smallest value of } \\
& \left(x_{1}, x_{2}, \cdots, x_{n}\right)
\end{aligned}
$$

and $x(a, b)$ is a literal operator defined as :

$$
x(a, b)= \begin{cases}m-1 & (a \leq x \leq b) \\ 0 & \text { (otherwise) }\end{cases}
$$

where $a$ and $b$ are window parameters of the literal function. Although there are many other forms, without loss of generality, we limit our discussions on the sum-ofproducts forms only. The proof of Equation (1) follows directly from the definition of fundamental functions. A canonical realization based on a sum-of-products expansion in which each input vector is individually selected, is always available, corresponding to the canonical sum-of-minterms realization of the binary case. In the $m$-valued $n$-variable case, $n \times\left(m^{n}-1\right)$ literal nodes are required in order that each input vector can be individually selected. Each of the literal operators $x_{i}(a, b)$, $i=1,2, \ldots, n$ and with appropriate $a$ and $b$ selects one (or more) column (row) of the function, giving these columns (rows) the value $(m-1)$, with 0 elsewhere. The general expansion would contain an $\left(m^{n}-1\right)$ product terms, thus corresponding to $\left(m^{n}-1\right) M I N$ nodes. Each product term therefore selects a particular area of the function, and defines its function value 1 or 2 or ... or $(m-1)$ by means of the logic signal 1 or 2 or ... or $(m-1)$ included within the product term. Finally, an $\left(m^{n}-1\right)$-input $M A X$ node is employed to give the summation of the $\left(m^{n}-1\right)$ product terms. As an example, consider a quaternary function shown in Table 1. In the quaternary case shown, the general expansion would contain a $4^{2}=16$ terms, five being zero-valued. The remaining eleven nonzero terms would read

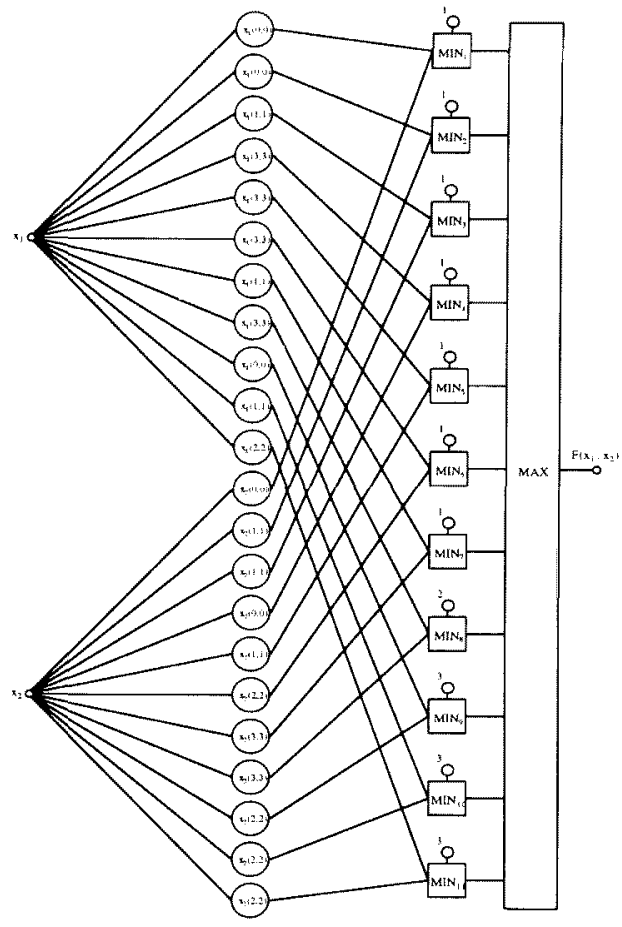

Fig. 1. A canonical realization based MVL architecture for the 4 -valued logic function of Table 1.

$$
\begin{aligned}
F\left(x_{1}, x_{2}\right)= & 1 \cdot x_{1}(0,0) \cdot x_{2}(0,0)+1 \cdot x_{1}(0,0) \cdot x_{2}(1,1)+1 \cdot x_{1}(1,1) \\
& \cdot x_{2}(1,1)+1 \cdot x_{1}(3,3) \cdot x_{2}(0,0)+1 \cdot x_{1}(3,3) \cdot x_{2}(1,1)+ \\
& 1 \cdot x_{1}(3,3) \cdot x_{2}(2,2)+1 \cdot x_{1}(1,1) \cdot x_{2}(3,3)+2 \cdot x_{1}(3,3) \\
& \cdot x_{2}(3,3)+3 \cdot x_{1}(0,0) \cdot x_{2}(2,2)+3 \cdot x_{1}(1,1) \cdot x_{2}(2,2)+ \\
& 3 \cdot x_{1}(2,2) \cdot x_{2}(2,2) \cdots \cdots \cdots \cdots \cdots \cdots
\end{aligned}
$$

The corresponding realization of Fig. 1 contains 22 literal nodes, $4^{2}-5=11 M I N$ nodes for five zero-valued terms and $4^{2}-5=11$ - input $M A X$ node. Several features may be noted in these realizations, including the followings:

a) The network with the literal, $M I N$ and $M A X$ nodes gives functional completeness, e.g., any multiplevalued logic function may be realized with the network

b) The realization gives detail informations on its network, such as how many layers are needed for a given task and how many units per layer, sometimes even the literal parameters, and other useful parameters.

\section{Learning MVL System}

We consider an $m$-valued logic system of $n$ inputs $x_{1}, x_{2}, \ldots, x_{n}$, and one output $F\left(x_{1}, x_{2}, \ldots, x_{n}\right)$. The corresponding architecture is shown in Fig.2, node functions in the same layer are of the same type, as described below:

Layer 1 : Each node in this layer is a literal function and its node function is given by

$$
x\left(a_{i j}, b_{i j}\right)= \begin{cases}m-1 & \left(a_{i j} \leq x \leq b_{i j}\right) \\ 0 & \text { (otherwise) }\end{cases}
$$




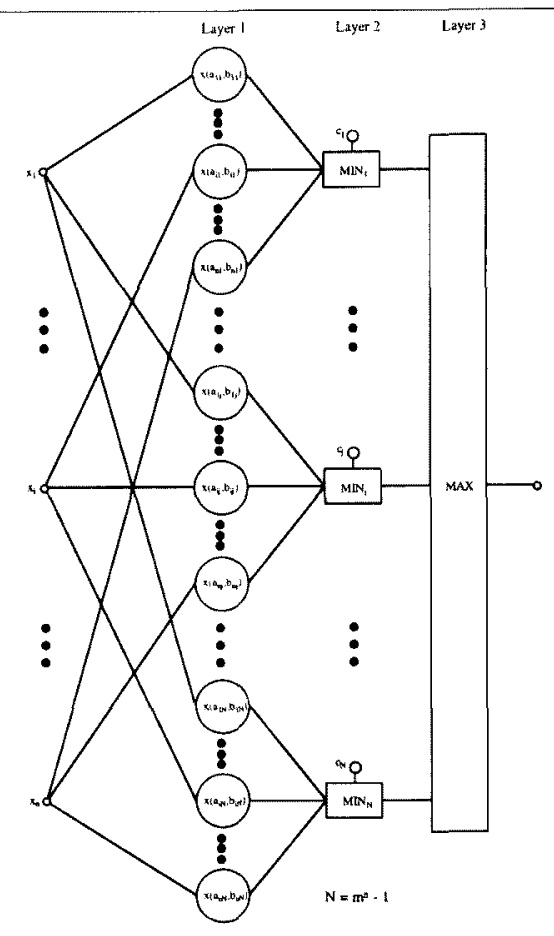

Fig. 2. A learning MVL architecture based on a canonical realization of MVL function.

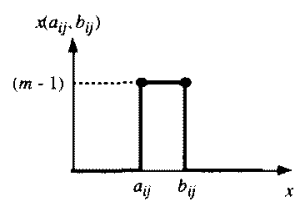

Fig. 3. The definition of a literal function.

where $a_{i j}, b_{i j}$ is a window parameter set and $a_{i j} \leq b_{i j}$ $\left(i=1,2, \cdots, n\right.$ and $\left.j=1,2, \cdots, m^{n}-1\right)$. The literal function for the node function is shown in Fig. 3. As the values of the $a_{i j}$ and $b_{i j}$ change, the literal function varies accordingly, thus exhibiting various form of literal functions. In an $m$-valued $n$-variable case, maximally $n \times$ $\left(m^{n}-1\right)$ literal nodes are required in order that each input vector can be individually selected. Although it is a very large number, in most cases, it can be greatly reduced in learning process as it can be done in logic reduction by MVL algebra. Furthermore, in real implementations, by introducing the one-input multipleoutput literal nodes, only $n \times m$ literal nodes will actually be necessary to represent any $n$-variable $m$-valued logic function. In order to do learning with gradient descent, derivatives of the function are needed. However, as shown in Fig. 3, the literal function shows a hard limiting nonlinearity with the window parameters $a_{i j}$ and $b_{i j}$. The derivatives of the literal function do not exist at the two points. As used in neural networks, the hard limiting nonlinearity can be replaced by a soft limiting function $f(h)$ called sigmoid function :

$$
f(h)=\frac{1}{1+\exp (-\lambda h)}
$$

when $\lambda \rightarrow \infty$. Thus, the literal function can be described as

$$
x\left(a_{i j}, b_{i j}\right)=(m-1)\left\{f\left(x-a_{i j}\right)-f\left(x-b_{i j}\right)\right\}
$$

where $a_{i j} \leq b_{i j}$. The function is clearly differential and saturates to 0 at both extremes. The derivative of the function can be expressed in terms of the function itself as :

$$
\frac{\partial x\left(a_{i j}, b_{i j}\right)}{\partial a_{i j}}=-(m-1) \lambda f\left(x-a_{i j}\right)\left\{1-f\left(x-a_{i j}\right)\right\}
$$

and

$$
\frac{\partial x\left(a_{i j}, b_{i j}\right)}{\partial b_{i j}}=(m-1) \lambda f\left(x-b_{i j}\right)\left\{1-f\left(x-b_{i j}\right)\right\}
$$

In the above discussions, we have made an approximation that the hard limiting nonlinearity can be computed by the sigmoid function. The formula (7) and (8) for derivatives of the literal function are very crude. We can therefore use only its sign, not its magnitude. Thus, the derivatives can be simply defined as

$$
\frac{\partial x\left(a_{i j}, b_{i j}\right)}{\partial a_{i j}}=-1
$$

and

$$
\frac{\partial x\left(a_{i j}, b_{i j}\right)}{\partial b_{i j}}=1
$$

Layer 2: A node in layer 2 corresponds to the $M I N$ operation. Each node selects a particular area of the function and defines its function value 1 or 2 , or $\cdots$, or $(m-1)$ by means of the logic signal 1 or 2 or $\cdots$, or $(m-1)$ included within the $M I N$ term. Thus, it corresponds to $\left(m^{n}-1\right) M I N$ nodes maximally. The function is given by

$$
\begin{aligned}
M I N_{j}= & M I N\left(c_{j}, x\left(a_{1 j}, b_{1 j}\right), \cdots, x\left(a_{i j}, b_{i j}\right), \cdots\right. \\
& \left.x\left(a_{n j}, b_{n j}\right)\right)
\end{aligned}
$$

where $c_{j}$ is biasing parameter of $M I N_{j}$ with the the logic signal 1 or 2 or $\cdots$ or $(m-1)$. In order to do learning with gradient descent, derivatives of the $M I N$ functions are also needed. However, the derivatives of the $M I N$ functions do not exist at the points where the right limit and left limit are not equal. The formally rigorous way to handle this is to consider the convex combination of all the gradients at the singular point, and to pick the one direction from this set that benefits the optimization algorithm most. A heuristic approximation to this scheme is to use an average of the two limits for the derivative at the singular point $[10]$. We chose the simpler heuristic approach. Thus, the derivatives of the $M I N$ function can be defined as : 


$$
\begin{aligned}
& \frac{\partial M I N_{j}}{\partial x\left(a_{i j}, b_{i j}\right)}= \\
& \left\{\begin{array}{cc}
1 & x\left(a_{i j}, b_{i j}\right)<M I N\left(c_{j}, x\left(a_{l j}, b_{i j}\right), l=1,2, \cdots, n, l \neq i\right) \\
\frac{1}{2} & x\left(a_{i j}, b_{i j}\right)=\operatorname{MIN}\left(c_{j}, x\left(a_{l j}, b_{i j}\right), l=1,2, \cdots, n, l \neq i\right) \\
0 & x\left(a_{i j}, b_{i j}\right)>\operatorname{MIN}\left(c_{j}, x\left(a_{i j}, b_{i j}\right), l=1,2, \cdots, n, l \neq i\right)
\end{array}\right.
\end{aligned}
$$

and

$$
\begin{aligned}
& \frac{\partial M I N_{j}}{\partial c_{j}}= \\
& \begin{cases}1 & c_{j}<M I N\left(x\left(a_{1 j}, b_{1 j}\right), \cdots x\left(a_{i j}, b_{i j}\right), \cdots x\left(a_{n j}, b_{n j}\right)\right) \\
\frac{1}{2} & c_{j}=M I N\left(x\left(a_{1 j}, b_{1 j}\right), \cdots x\left(a_{i j}, b_{i j}\right), \cdots x\left(a_{n j}, b_{r j}\right)\right) \\
0 & c_{j}>M I N\left(x\left(a_{1 j}, b_{1 j}\right), \cdots x\left(a_{i j}, b_{i j}\right), \cdots x\left(a_{n j}, b_{n j}\right)\right)\end{cases}
\end{aligned}
$$

Layer 3 : This node gives an $M A X$ operator between the product terms :

$$
O=M A X\left(M I N_{1}, M I N_{2}, \cdots, M I N_{\left(m^{n}-1\right)}\right)
$$

Its derivative can also be written as :

$$
\begin{aligned}
& \frac{\partial M A X}{\partial M I N_{j}}= \\
& \begin{cases}1 & M I N_{j}<M A X\left(M I N_{k}, k=1,2, \cdots,\left(m^{n}-1\right), k \neq j\right) \\
\frac{1}{2} & M I N_{j}=M A X\left(M I N_{k}, k=1,2, \cdots,\left(m^{n}-1\right), k \neq j\right) \\
0 & M I N_{j}>M A X\left(M I N_{k}, k=1,2, \cdots,\left(m^{n}-1\right), k \neq j\right)\end{cases}
\end{aligned}
$$

Of course, a soft $M I N$ function and a soft $M A X$ function can be used for the differentiability ${ }^{[14]}$. The learning MVL network described above is a multilayered feedforward network in which each node performs a particular function (node function) on incoming signals using a set of parameters specific to this node. The form of node function varies from layer to layer, and each node function can be defined by prior knowledge on the network. Unlike the traditional neural networks, the MVL networks give the maximal numbers of the nodes needed for any MVL functions. Furthermore we can use the prior knowledge on MVL functions to initialize the nodes' parameters if we have, even sometimes they are not complete. Thus, the learning can start from a relatively "good" point.

\section{Learning Mechanism}

The learning MVL network mentioned above is a feedforward network. Let $\Theta$ be a set containing pairs of sampled multiple-valued input vectors $\overrightarrow{X^{\prime}} s$, and the corresponding teacher's signal vectors $\overrightarrow{T^{\prime}} s$ generated by a given multiple-valued function. Using a similar method as back-propagation algorithm, the parameters $\left(a_{i j}, b_{i j}\right.$ and $c_{j}$ ) are modified so as to minimize a cost function of the form :

$$
E=\sum_{(\vec{X}, \vec{T}) \in \Theta}(\vec{O}-\vec{T})^{2}
$$

where $\vec{O}$ is the output vector (at the output layer) of the network when input vector $\vec{X}$ is applied at the input. Back propagation employs gradient descent to minimize $E$. That is, the parameters $\left(a_{i j}, b_{i j}\right.$ and $\left.c_{j}\right)$ are varied in accordance with the rules:

$$
\begin{aligned}
& \Delta a_{i j}=-\eta \frac{\partial E}{\partial a_{i j}} \\
& \Delta b_{i j}=-\eta \frac{\partial E}{\partial b_{i j}}
\end{aligned}
$$

and

$$
\Delta c_{j}=-\eta \frac{\partial E}{\partial c_{j}}
$$

where $a_{i j}$ and $b_{i j}$ are the literal parameters, $c_{j}$ is the biasing parameter of $M I N_{j}$ and $\eta(0<\eta<1)$ is the learning rate respectivily. For the parameters $\Delta a_{i j}$, $\Delta b_{i j}$ and $\Delta c_{j}$, we must differentiate with respect to the $a_{i j}, b_{i j}$ and $c_{j}$ which are deeply embedded in (14). Using the chain rule, we obtain :

$$
\begin{aligned}
\Delta a_{i j}= & -\eta \frac{\partial E}{\partial a_{i j}}=-\eta \sum \frac{\partial E}{\partial M \vec{A} X} \cdot \frac{\partial M \vec{A} X}{\partial a_{i j}} \\
= & -\eta \sum(\vec{T}-M \vec{A} X) \frac{\partial M \vec{A} X}{\partial M \vec{I} N_{j}} \cdot \frac{\partial M \vec{I} N_{j}}{\partial a_{i j}} \\
= & -\eta \sum(\vec{T}-M \vec{A} X) \frac{\partial M \vec{A} X}{\partial M \vec{I} N_{j}} \cdot \frac{\partial M \vec{I} N_{j}}{\partial x\left(a_{i j}, b_{i j}\right)} \\
& \cdot \frac{\partial x\left(a_{i j}, b_{i j}\right)}{\partial a_{i j}} \\
= & -\eta \sum(\vec{T}-M \vec{A} X) \frac{\partial M \vec{A} X}{\partial M \vec{I} N_{j}} \cdot \frac{\partial M \vec{I} N_{j}}{\partial x\left(a_{i j}, b_{i j}\right)} \\
\Delta b_{i j}= & \eta \sum(\vec{T}-M \vec{A} X) \frac{\partial M \vec{A} X}{\partial M \vec{I} N_{j}} \cdot \frac{\partial M \vec{I} N_{j}}{\partial x\left(a_{i j}, b_{i j}\right)}(17)
\end{aligned}
$$

and

$$
\Delta c_{j}=-\eta \sum(\vec{T}-M \vec{A} X) \frac{\partial M \vec{A} X}{\partial M \vec{I} N_{j}} \cdot \frac{\partial M \vec{I} N_{j}}{\partial c_{j}}(18)
$$

Using the above learning equations and the derivatives defined above, we can carry out the back-propagation to the MVL networks.

\section{Reduction, Learning and Cytology Behav- iors}

We begin this discussion by recalling a few fundamental techniques and conventions found in multiple-valued logic. An MVL function can be represented in several ways and in various domain. Three of these representations are the algebraic expression, the truth table, and 
Table 2. Example 2 of a quaternary function.
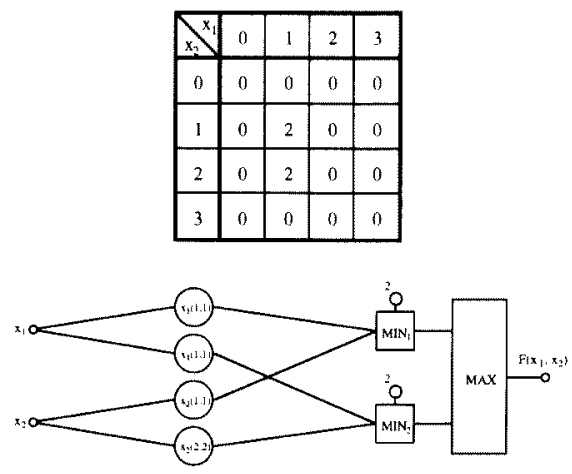

(a)

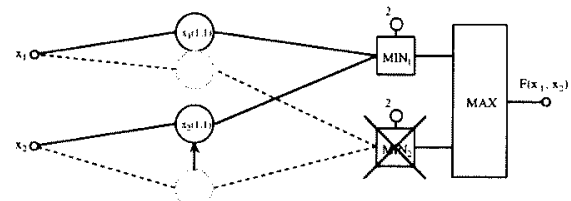

(b)

Fig. 4. The reduction process of the 4-valued logic function of Table 2 .

Table 3. Example 3 of a quaternary function.

\begin{tabular}{|c|c|c|c|c|}
\hline$x_{2}$ & 0 & 1 & 2 & 3 \\
\hline 0 & 0 & 2 & 0 & 0 \\
\hline 1 & 0 & 2 & 0 & 0 \\
\hline 2 & 0 & 2 & 0 & 0 \\
\hline 3 & 0 & 2 & 0 & 0 \\
\hline
\end{tabular}

the Karnaugh map. These will be subsequently used as aids to help explain the operation of the learning MVL networks. An MVL algebraic function of logic variables can be used to represent a multiple-input-single-output logic function. For example, if we have a two-input quaternary function of Table 2, we can write

$$
F\left(x_{1}, x_{2}\right)=2 x_{1}(1,1) \cdot x_{2}(1,1)+2 x_{1}(1,1) \cdot x_{2}(2,2)
$$

A corresponding network realization is shown in Fig. 4(a). The use of MVL algebra allows us to rewrite (19) to the form of $(20)$

$$
F\left(x_{1}, x_{2}\right)=x_{1}(1,1)\left(2 x_{2}(1,1)+2 x_{2}(2,2)\right)
$$

Further, we can perform logical reduction by basic MVL algebra and obtain

$$
F\left(x_{1}, x_{2}\right)=2 x_{1}(1,1) \cdot x_{2}(1,2) \ldots \ldots \ldots \ldots
$$

The above reduction can be viewed as requiring only one $M I N$ term to implement $2 x_{1}(1,1) x_{2}(1,2)$. Thus, we have realized a reduction of two $M I N$ terms to one $M I N$ term. The corresponding realizations after the reduction is shown in Fig. 4(b). The former operation (Eq. (20)) can be viewed as the simple cell adhesion that dose not change any cell's properties, and the latter one (Eq. (21)) can be represented as the complex cell adhesion that produces new cell's properties, which corresponds to the overlap of the function areas or the absorption.

We now refer to Eqs. (20) and (21) for an example to discuss the operations of the $x_{1}(1,1)$ and $x_{2}(2,2)$ cells between inputs and the $M I N_{2}$ cell. Under the reduction of Eq. (20) to (21), the prior presentation of the $M I N_{1}$ term $2 x_{1}(1,1) x_{2}(1,1)$ as belonging to function $F\left(x_{1}, x_{2}\right)$ would have already resulted in the determination of $2 x_{1}(1,1) x_{2}(1,2)$ as being part of function $F\left(x_{1}, x_{2}\right)$ with the $x_{1}(1,1)$ cell and the $x_{2}(2,2)$ cell adhere to the $x_{1}(1,1)$ cell and the $x_{2}(1,2)$ cell, respectively. But,the $M I N_{2}$ term $2 x_{1}(1,1) x_{2}(2,2)$ has been eliminated in the reduction procedure, which can be viewed as the aptopsis in cytology. This is accomplished by setting the window parameters $a(\leq b)$ of the cells between the inputs and the $M I N_{2}$ cell to be larger than 3 , thus zeroing at least one input to the $M I N_{2}$ cell, for example $x_{1}(1,1)$ between $x_{1}$ and $M I N_{2}$ to be $x_{1}(4,4)$ or $x_{2}(2,2)$ between $x_{2}$ and $M I N_{2}$ to be $x_{2}(4,4)$ or both. This will result in $M I N_{2}=0$. Thus, the $M I N_{2}$ term is overlapped because of the $M A X$ operator between the $M I N$ cell terms. This corresponds to the $M I N$ cell aptosis.

As a specific example, consider the Karnaugh map of Table 3 and the associated algebraic equation

$$
\begin{aligned}
F\left(x_{1}, x_{2}\right)= & 2 x_{1}(1,1) \cdot x_{2}(0,0)+2 x_{1}(1,1) \\
& x_{2}(1,1)+2 x_{1}(1,1) \cdot x_{2}(2,2)+ \\
& 2 x_{1}(1,1) \cdot x_{2}(3,3) \cdots \ldots \ldots
\end{aligned}
$$

We can perform logic reduction and obtain

$$
F\left(x_{1}, x_{2}\right)=2 x_{1}(1,1)
$$

The above reduction can be viewed as requiring only one $M I N$ cell to implement the $2 x_{1}(1,1) x_{2}(0,0)$. Further, the $M I N$ term requires only $2 x_{1}(1,1)$ and hence the $x_{2}(0,0)$ is eliminated from the term. This is accomplished by setting the window parameter $a$ of the $x_{2}(0,0)$ to be smaller than 0 and $b$ larger than 3 , thus resulting in a constant 3 input to the $M I N_{1}$ cell. Thus, the cell is overlapped because of the $M I N$ operation between the literal cells. This can be viewed as the literal cell aptopsis, as shown in Fig. 5. Further, if one of the $M I N$ cells is biased to be a constant 3 , all $M I N$ cells and $M A X$ cells can be eliminated and the network outputs a constant 3 . This corresponding to the cluster cell aptosis. Therefore, during the learning, the modification of the window parameters may lead to the simple and complex cell adhesions, corresponding to the distributivity and the absorption in MVL algebra. The modification may also cause the literal cell aptosis, the $M I N$ cell aptosis and the cluster cell aptosis. They correspond to the literal cell not including in the $M I N$ 


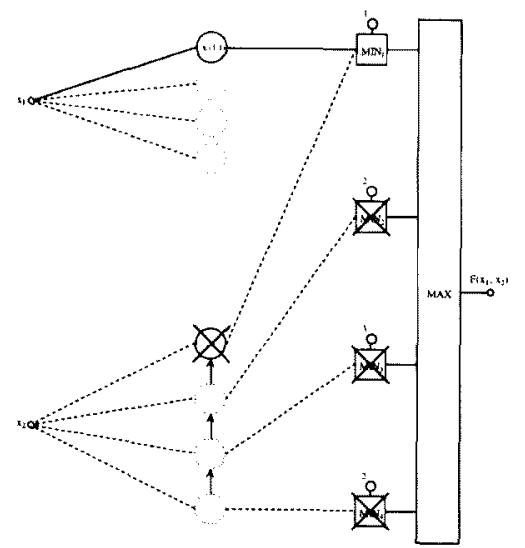

Fig. 5. The reduction process of the 4-valued logic function of Table 3 .

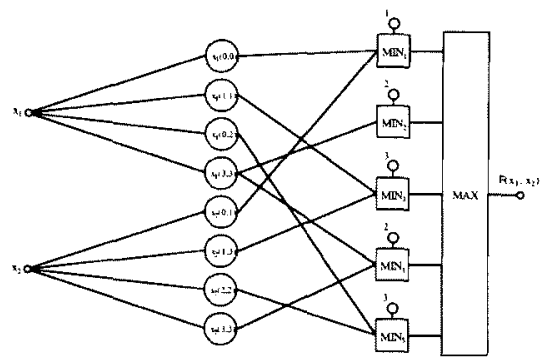

Fig. 6. A possible minimized realization for the function of Table 1

term and outputting a constant 3 , the $M I N$ term being biased in a constant 0 and not including in the sumof-products form, and all cells dying and outputting a constant 3 , respectively. We have shown that the logic reductions can account for some cytology behaviors. A discussion of the implementation of the reduction on the example given in Section 2 using the data of Table 1 will now be conducted. We begin with the default MVL network depicted in Fig. 1 having 22 literal cells, $11 M I N$ cells and one $M A X$ cell. Because of the $M A X$ cell between product terms ( $M I N$ terms), any higher-valued product term may overlap a lower-valued product term if advantageous, e.g., see Table 4(c) and (e) and also (d) and (f). Thus, one possible synthesis for this function can be the summation of five terms, as follows:

$$
\begin{aligned}
F\left(x_{1}, x_{2}\right)= & 1 x_{1}(0,0) \cdot x_{2}(0,1)+1 x_{1}(3,3)+ \\
& 1 x_{1}(1,1) \cdot x_{2}(1,3)+2 x_{1}(3,3) \\
& \cdot x_{2}(3,3)+3 x_{1}(0,2) \cdot x_{2}(2,2) \cdots
\end{aligned}
$$

where the logic signal " 3 " in the last terms is redundant. Thus, we have realized a reduction of 22 literal cells to 9, and $11 M I N$ cells to 5, as shown in Fig. 6 . Thus, adjusting the width of the literal window and the biasing parameters with the back-propagation may produce a minimized realization. Therefore, before learning we can initialize the MVL network to a small or a very
Table 4. Example 4 of a quaternary function,
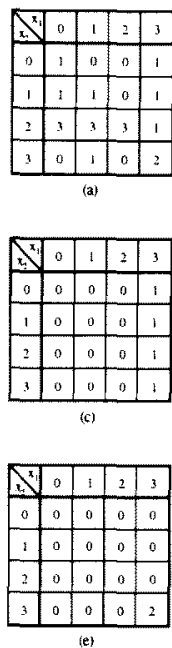

(e)
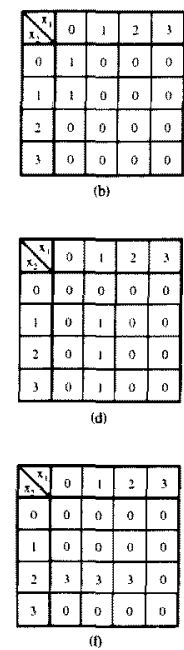

small one by our prior knowledge on MVL functions and reduction algebra. If it doesn't yield an acceptable solution, we can increase the size of the network, for example, the numbers of literal windows and $M I N S$, and train it until an acceptable convergent solution is found.

\section{Simulation Results}

We will now give several examples of the learning MVL networks to demonstrate their applicability. Two kinds of simulations were conducted, one in which the incomplete inputs/outputs or incompletely correct inputs/outputs information was employed, and one in which the completely unknown inputs/outputs information was used. Consider a simple example of a twovariables $\left(x_{1}, x_{2}\right)$, 4-valued function shown in Fig. 7(a). A canonical realization of the function can be the summation of the three terms:

$$
\begin{aligned}
F\left(x_{1}, x_{2}\right)= & 1 x_{1}(0,0) \cdot x_{2}(0,0)+2 x_{1}(0,0) \\
& \cdot x_{2}(3,3)+3 x_{1}(0,0) \cdot x_{2}(2,2) .
\end{aligned}
$$

The canonical realization clearly shows an MVL architecture-the topology and the number of hidden layers and units for the function. Fig. 8(a) illustrates the MVL network and learning is performed on the network. In the simulations, for simplicity, the training is conducted in a manner analogous to neural backpropagation and only limited on literal window parameters, although learning with biasing parameters $c_{j}$ is more effective and nature. The prior knowledge from the canonical realization can be used as the initial parameters of the learning process. Thus, the learning can get started from a point in the parameter space that is not far from the optimal. In the learning MVL system, the literal window $\left(a_{i j}, b_{i j}\right)$ determines how much an input affects the final output of the network. The modification of the literal window corresponds to the modification of the area of the function. In the first experiment, we use Fig. $7(\mathrm{a})$ as the teacher's signals and 


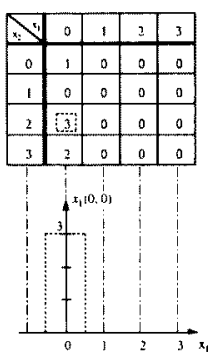

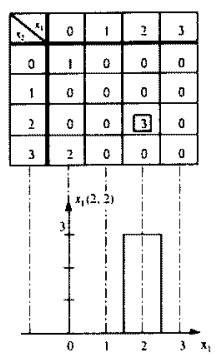

(b)
Fig. 7. Learning to shift an inappropriate literal window function: (a) before and (b) after learning.
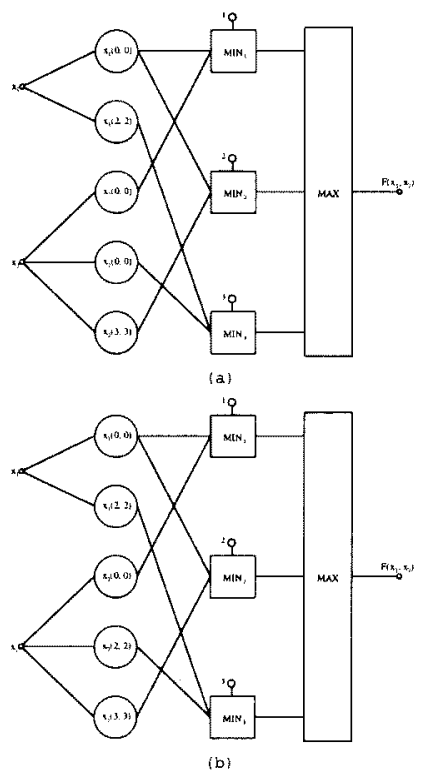

Fig. 8. A canonical realization based MVL architectuve for the 4-valued logic function (a) before and (b) after learning.

show how the learning can shift the literal window from $x_{1}(0,0)$ to $x_{1}(2,2)$. Simulation shows that the system learned to shift it to the correct place $x_{1}(2,2)$, as shown in Fig. 7(b) and Fig. 8(b).

Furthermore, in training an MVL network through the literal window $\left(a_{i j}, b_{i j}\right)$, adjusting the term set means widening or narrowing individual windows, depending on whether the previous response was below or above the expected valued. If the system response in the preceding model execution was above the expected or desired valued, then for each literal window in the term set that was accessed during that execution, the domain is slightly narrowed. That is, the left edge of the domain is moved slightly to right and the right edge is moved slightly to left. In the second experiment, we first initialized the literal parameter of $x_{1}$ to cover an area from 0 to 2, as shown in Fig. 9(a) and trained the MVL network with an MVL function defined in Fig. 9(b). The

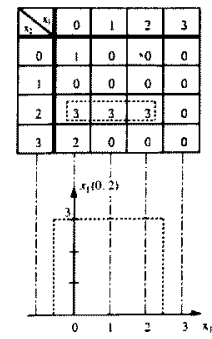

(a)

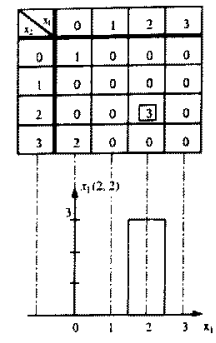

Fig. 9. Learning to narrow an inappropriate literal window function : (a) before and (b) after learning.

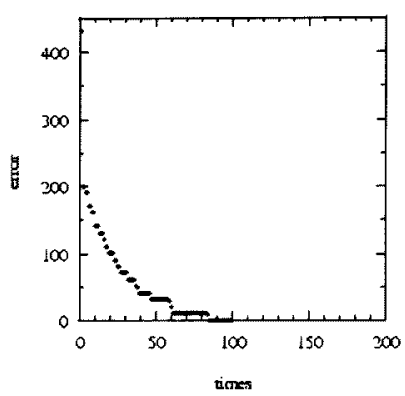

Fig. 10. Learning curve for the 4-valued logic function of Fig. 9

learning characteristics is shown in Fig. 10. We see that after learning, the literal function narrowed to a correct width. Conversely, if the system response was below expectation, the involved domains are slightly widened - their left edges are moved slightly to the right. In the third simulation, we initialized the literal function parameter with $x_{1}(1,1)$ as shown in Fig. 11(a) and then force the MVL network to yield an output as shown in Fig. 11(b). Fig. 12 shows the learning characteristics. It can be seen that after a few iterations, the literal function was widen to cover the area from 0 to 2 , indicating the MVL networks are able to learn very flexibly. Generally speaking, when the literal window is widened, then the contribution of the input is increased, when the literal window is narrowed, the contribution of the input is decreased.

In all simulations above, we used the incomplete or incompletely correct inputs/outputs information. Now we discuss the completely inputs/outputs completely unknow problem. For an inputs/outputs unknown problem, the selection of an architecture with appropriate size is mainly empirical. For the most part, different configurations (for example, the numbers of $M I N s$ and literal windows) are tried, and if they do not yield an acceptable solution, they are discarded. Another topology is then defined and the whole training process is repeated. We consider a 2 -variable 4 -valued inputs/outputs unknown problem. By our prior knowledge on MVL function, the problem needs at least 3 


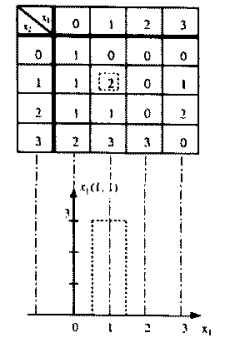

(a)

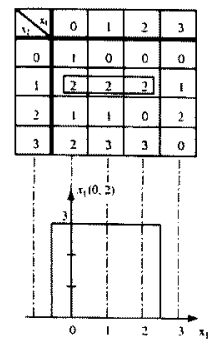

(b)
Fig. 11. Learning to wide an inappropriate literal window function : (a) before and (b) after learning.

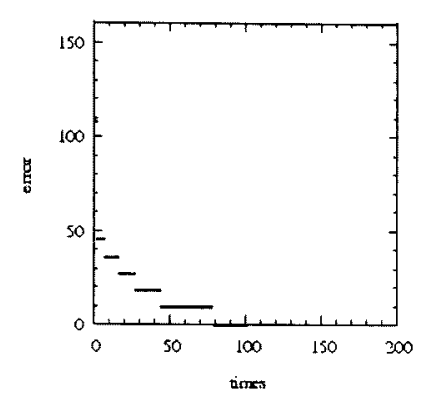

Fig. 12. Learning curve for the 4-valued logic function of Fig. 11.

Table 5. Example 5 of a quaternary function.

\begin{tabular}{|c|c|c|c|c|}
\hline$x_{3}$ & 0 & 1 & 2 & 3 \\
\hline 0 & 1 & 0 & 0 & 0 \\
\hline 1 & 0 & 0 & 0 & 0 \\
\hline 2 & 0 & 0 & 3 & 0 \\
\hline 3 & 2 & 0 & 0 & 0 \\
\hline
\end{tabular}

$M I N s$ biased in 1,2 and 3 to provide an output with $0,1,2$ or 3 . Thus, the 2 variable requires maximally 6 literal nodes. The network is shown in Fig. 13 and learning is performed on the network. Then, we initialized the all literal parameters of the network of Fig. 13(a) from 0 to 4 randomly. The learning rate for these literal parameters is fixed at 0.01 . Suppose we have a 2-variable 4 -valued training set shown in Table 5. Fig. 14 shows the learning curve for the 4-valued problem. After learning for about 168 times, the error decreased from 152 to 0 . Fig. 13(b) shows the network after learning. It is obvious that the learning MVL network provides a good learning characteristics. Furthermore, by logical reduction or cytology behaviors, the network of Fig. 13(b) can be reduced to the network as shown in Fig. 13(c). However it should be noted that the learning doesn't always work so well and it depends strongly on initial parameters. Further, it is difficult or impossible for the network of Fig. 13(a) to learn a more complex problem and it typically requires more $M I N S$ and literal nodes.

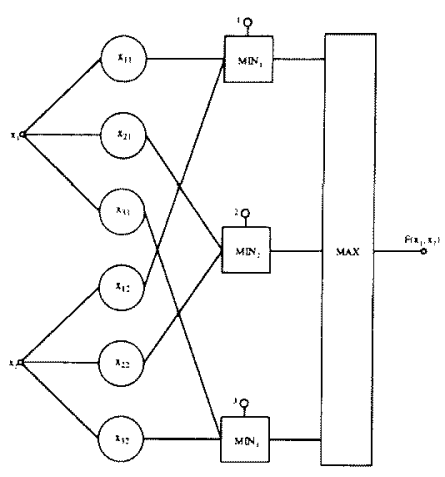

(a)

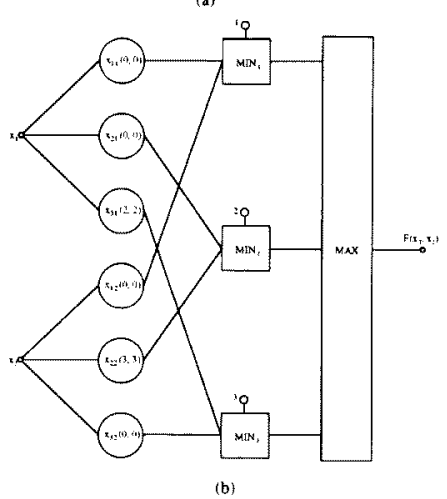

(b)

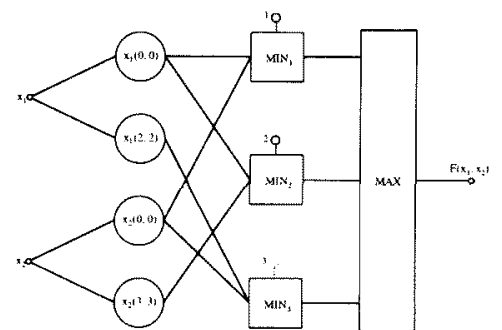

(c)

Fig. 13. Learning MVL network : (a) initialized (before learning) (b) after learning and (c) after logical reduction or cytology operation.

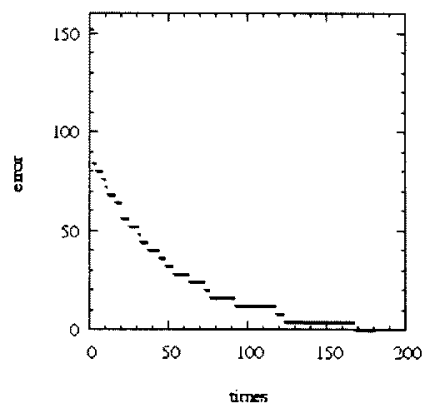

Fig. 14. Learning curve for the 4-valued logic function of Table 5 .

\section{CONCLUSIONS}

A learning MVL network based on back-propagation 
is introduced. The learning MVL systems usually work like back-propagation neural network, by examining a solution with a target result. But unlike neural networks, the learning MVL systems are more likely to run with a prior knowledge base. Neural networks require arbitrary choices regarding the number of hidden units and layers. The learning occurs in a "black box" without any prior knowledge about the architecture. The learning MVL networks are based on the canonical realizations of multiple-valued logic functions. The functional completeness of the network is guaranteed and any multiple-valued logic functions can be realized with the networks. Furthermore, the canonical realization gives the architecture - the topology, the number of hidden layers and units, sometimes even literal parameters and other useful parameters. The prior knowledge can be used as the initial parameters of the learning MVL network in the learning process. Thus, the learning process can get started from a point in the parameter space that is not far from the optimal one. It is obviously useful in speeding up the convergence and avoiding local minima. Furthermore, unlike neural networks, each node in the learning MVL network performs a particular function (node function) on incoming signals using a set of parameters specific to this node. The form of node function may vary from node to node. Some are modifiable, and some are unmodifiable. Examples are provided that demonstrate the ability of the learning MVL to find reasonable literal windows, and learn MVL problems. The learning MVL networks simulated some cytology behaviors, such as the cell adhesions, the cell aptosis and the cluster cell aptopsis successfully.

\section{Acknowledgement}

This work was supported in part by the International Communications Foundation. The authors would also like to thank the reviewers, whose comments made useful contribution to the quality of this paper.

(Manuscript received December 28, 1998, revised April 26, 1999)

\section{References}

(1) M. Kameyama, T. Hanyu and T. Higuchi, "Design and implementation of quaternary NMOS integrated circuits for pipeline image processing," IEEE J. Solid-State circuits, vol. SC12 , no.1, pp. 20-27, 1987

(2) K. C. Smith, "A multiple-valued logic: A tutorial and application," Computer, vol. 21, no. 4, pp.17-27, 1988.

(3) Z. Tang, O. Ishizuka, Q. Cao and H. Matsumoto, "Algebraic properties of a learning multiple-valued logic network," in Proc. 23rd IEEE International Symposium on MultipleValued Logic, Sacramento, California, May, 1993, pp.196-201.

(4) Q. Cao, O. Ishizuka, Z. Tang and H. Matsumoto, "Algorithm and implementation of a learning multiple-valued logic network," in Proc. 23rd IEEE International Symposium on Multiple-Valued Logic, Sacramento, California, May, 1993, pp.202-207.

(5) Z. Tang, O. Ishizuka and Q. Cao, "A learning multiple-valued logic network: algebra, algorithm and applications," IEEE Trans. on Computer, vol.47, no.2, pp.247-251, 1998

(6) T. Watanabe, et. al., "A design of multiple-valued logic neuron," in Proc. 20th IEEE International Symposium on Multiple-Valued Logic, Charlotte, North Carolina, May, 1990 pp.418-426.

(7) T. Watanabe et. al., "Layered MVL neural networks capable of recognizing translated characters," in Proc. 22th IEEE International Symposium on Multiple-Valued Logic, Sendai, Japan, May, 1992, pp.88-95.

(8) Y. Hata, T.Hozumi and K. Yamato, "Gate model networks for minimization of multiple-valued logic functions," in Proc. 23th IEEE International Symposium on MultipleValued Logic, Sacramento, Califonia, May, 1993, pp.29-34.

(9) E. L. Post, "Introduction to general theory of elementary propositions ", Amer J. Math., vol.43, pp.163-185,1921.

(10) E. Muhldorf, "Schaltungen fur ternare Schaltvariable", Arch Elekt. Ubertrag., vol.12, pp.176-182,1959.

(11) M. Yoeli and G. Rosenfield, "Logic design of switching circuits", IEEE Trans. Electron. Comput., vol. EC-14, pp.1929,1965 .

(12) C. M. Allen and D. P. Givone, "A minimization technique for multiple-valued logic systems," IEEE Trans. on Comput. vol. C-17, pp.182-184, 1968.

(13) S. Y. Su and A. A. Sarris, "The relationship between multivalued switching algebra and Boolean algebra under different definitions of complement," IEEE Trans. on Comput., vol.C21, pp.479-485, 1972.

(14) H. R. Berenji and P. Khedkar, "Learning and tuning fuzzy logic controllers through reinforcements, "IEEE Trans. on Neural Networks, vol. 3, no. 5, pp.724-740, 1992.

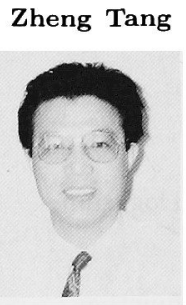

(Non-member) He was born in 1959. He re ceived the B.S. degree from Zhejiang University, Zhejiang, China in 1982 and the M.S. degree and the Ph.D. degree from Tsinghua University, Beijing, China in 1984 and 1988 respectively. From 1988 to 1989 , he was Instructor in Institute of Microelectronics at Tsinghua University. In 1989, he joined Miyazaki University, Miyazaki, Japan, where he is currently Associate Professor in Department of Electrical \& Electronic Engineering. His current research interests include neural networks, multiple-valued logic and analog integrated circuit design.

Okihiko Ishizuka (Non-member) $\mathrm{He}$ was born in 1939

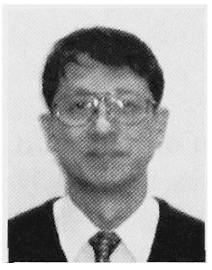
$\mathrm{He}$ received the B.E. degree in 1964 from Kagoshima University, and the M.E. degree in 1966 and the D.E. degree in 1978 from Kyusyu University. He is a Professor of Electrical \& Electronic Engineering, Miyazaki University, Miyazaki, Japan. His research interests are network synthesis, and circuit analysis on multi-valued logic, threshold logic and fuzzy logic. Dr.Ishizuka is a member of IEEE and Executive Subcommittee of Computer Society Technical Committee on Multi-Valued Logic.

Koichi Tanno (Non-member) He was born in 1967. He re-

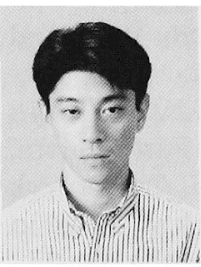
ceived the B.S. and M.S. degrees in Faculty of Engeering from Miyazaki University, in 1990 and 1992, respectively. From 1992 to 1993 , he joined the Microelectronics Products Development Laboraroty, Hitachi, Ltd., Yokohama, Japan. He has been engaged in research in the low supply voltage continuous-time filter. Since 1994, he has been with the Faculty of Engineering, Miyazaki University where he is now a Research Associate in the Department of Electrical \& Electronic Engineering. His main research interests are bipolar and CMOS analog circuits and hardware realization of fuzzy controller and neural networks. Mr. Tanno is a member of IEEE. 